\title{
EQUAÇÕES HIPSOMÉTRICAS, VOLUMÉTRICAS E DE TAPER PARA ONZE ESPÉCIES NATIVAS ${ }^{1}$
}

\author{
Carlos Pedro Boechat Soares², Fabrina Bolzan Martins ${ }^{3}$, Heitor Urias Leite Junior ${ }^{4}$, \\ Gilson Fernandes da Silva ${ }^{5}$ e Lívia Thaís Moreira de Figueiredo ${ }^{6}$
}

\begin{abstract}
RESUMO - Este trabalho teve como objetivo ajustar equações hipsométricas, volumétricas e de taper para onze espécies florestais plantadas em parcelas experimentais, localizadas no campus da Universidade Federal de Viçosa (MG). Após análises, verificou-se que: a) o modelo hipsométrico 2 ajustou-se melhor às nove espécies; b) o modelo volumétrico 2 ajustou-se melhor aos dados de volume do fuste com casca para seis espécies, enquanto o modelo volumétrico 1 ajustou-se melhor para cinco espécies; c) o modelo de taper 3 foi superior aos demais modelos, sendo selecionado para descrever o perfil do tronco de dez espécies.
\end{abstract}

Palavras-chave: Equações alométricas, Espécies nativas brasileiras e Mata Atlântica.

\section{HIPSOMETRIC, VOLUMETRIC AND TAPER EQUATIONS FOR ELEVEN BRAZILIAN NATIVE SPECIES}

\begin{abstract}
The objective of this study was to adjust hypsometric, volumetric and taper equations for eleven native species planted in experimental plots, at Universidade Federal de Viçosa, Minas Gerais, Brazil. After analysis, it was verified that: a) the hipsometric model 2 best adjusted for nine species; $b$ ) the volumetric model 2 was best adjusted to the data of main stem volume with bark for six species, while the volumetric model was better adjusted for five species; c) Taper model 3 showed better performance, being selected to describe the profile stem of ten species.
\end{abstract}

Keywords: Alometric equations, Brazilian native species and Atlantic Rainforest.

\section{INTRODUÇÃO}

As equações hipsométricas, volumétricas e de taper (ou afilamento) são imprescindíveis no inventário florestal, pois permitem a totalização do volume por unidade de área para determinado fim.

No Brasil, várias equações de volume foram ajustadas para espécies de rápido crescimento do gênero Eucalyptus e Pinus, com destaque para o uso do modelo de Schumacher e Hall (1933) e da variável combinada (SPURR, 1952), conforme citado por Batista et al. (2004).
Equações de volume também foram ajustadas para diferentes biomas brasileiros, sem, contudo, possuírem especificidade para cada espécie (FERNANDES et al., 1983; SILVA; CARVALHO, 1984; PINHEIRO et al., 1985; ZAKIA et al., 1990; SOUZA; JESUS, 1991; CETEC, 1995).

Considerando a diversidade de espécies que compõem os biomas brasileiros, bem como a diversidade de tamanhos das árvores, equações de volume deveriam ser ajustadas para espécies individualmente ou para grupos de espécies com características semelhantes para aumentar a exatidão das estimativas volumétricas.

\footnotetext{
${ }^{1}$ Recebido em 26.04.2010 e aceito para publicação em 02.05.2011.

${ }^{2}$ Departamento de Engenharia Florestal, Universidade Federal de Viçosa - UFV, MG, Brasil. E-mail: <csoares@ufv.br>.

${ }^{3}$ Intituro de Recursos Naturais, Universidade Federal de Itajubá - UNIFEI, MG, Brasil. E-mail: <fabrinabm@gmail.com>

${ }^{4}$ Engenheiro Florestal. E-mail: <heitor_urias@hotmail.com>.

${ }^{5}$ Departamento de Engenharia Florestal, Universidade Federal do Espírito Santo, UFES, ES, Brasil. E-mail: <gfsilva2000@yahoo.com.br>.

${ }^{6}$ Programa de Pós-graduação em Ciência Florestal, Universidade Federal de Viçosa-UFV, MG, Brasil. E-mail: $<$ livia.figueiredo@hotmail.com>.
} 
Nesse sentido, poucos trabalhos foram realizados, como os de Scolforo et al. (1993) com angico (Anadenanthera macrocarpa), aroeira (Myracrydryon urundeuva) e jatobá (Hymeneaea courbaril); Scolforo et al. (1994) com pindaíba (Xylopia brasiliensis), canela (Ocotea odorifera) e copaíba (Copaifera langsdorffii); Batista et al. (2004) com caxeta (Tabebuia cassinoides); Scolforo et al. (2004) com candeia (Eremanthus erytropappus).

Nos trabalhos citados anteriormente, as equações foram ajustadas para espécies em condições naturais de ocorrência. Poucos são os estudos nos quais equações de volume foram ajustadas para espécies nativas brasileiras em plantios homogêneos, como os de Yared et al., (1993) com morototó (Schefflera morototonii); Araujo et al. (2004) com jurema-preta (Mimosa tenuiflora) e Tonini et al. (2005) com andiroba (Carapa guianensis), castanha-do-brasil (Bertholletia excelsa), ipê-roxo (Tabebuia avellanedae) e jatobá (Hymeneaea courbaril).

A altura de uma árvore é uma variável difícil de obter em florestas naturais densas e de dossel superior elevado. Como alternativa para solucionar este problema, tem-se a utilização de equações hipsométricas, que permitem estimar a altura por meio do seu diâmetro à altura do peito - DAP (SOARES et al., 2006). Este é um procedimento usual para florestas plantadas com espécies de rápido crescimento.

Devido à dificuldade de estimar a altura das árvores e à não utilização de equações hipsométricas, equações de volume têm sido ajustadas para florestas nativas, tendo como variável independente apenas o DAP (SILVA; ARAÚJO, 1984). Do ponto de vista estatístico, estas equações possuem erro de especificação (GUJARATI, 2000), uma vez que o volume do fuste das árvores depende da sua altura, gerando, portanto, estimativas tendenciosas. Árvores com mesmo diâmetro (DAP) podem possuir alturas diferentes e diversas formas dos fustes e, consequentemente, volumes variados.

As equações de taper ou afilamento foram muito bem estudadas para descrever o perfil de espécies de rápido crescimento no Brasil (LIMA, 1986; SILVA, 1996; ASSIS, 2002). Para florestas nativas brasileiras, há apenas o trabalho de Chichorro et al., (2003) para quantificar múltiplos produtos da madeira em floresta atlântica.
Pelo exposto, observa-se que ênfase tem sido dada ao desenvolvimento e ao ajuste de equações hipsométricas, volumétricas e de taper para espécies exóticas de rápido crescimento, dada a importância econômica dessas espécies. Contudo, as florestas naturais brasileiras apresentam muitas espécies de alto valor comercial, de tal forma que estudos desta natureza devam ser desenvolvidos para aprimorar as técnicas e os métodos de mensuração para florestas tropicais.

Este trabalho teve por objetivo ajustar equações hipsométricas volumétricas e de taper para 11 espécies nativas brasileiras, plantadas em parcelas experimentais, com o intuito de contribuir para o desenvolvimento da dendrometria dessas espécies e incentivar novos estudos em espécies nativas do Brasil.

\section{MATERIAL E MÉTODOS}

\subsection{Caracterização da área de estudo}

Este estudo foi desenvolvido na área denominada Mata da Silvicultura, situada no campus da Universidade Federal de Viçosa, localizada no Município de Viçosa, Minas Gerais ( $20^{\circ} 45^{\prime}$ de latitude e $42^{\circ} 55^{\prime}$ ' de longitude oeste). A área é classificada como Floresta Estacional Semidecidual Montana. Inclui-se no domínio da Mata Atlântica secundária e atualmente encontra-se em estágio médio de sucessão florestal (MEIRA NETO; MARTINS, 2000).

A precipitação média anual da região é de aproximadamente $1221,4 \mathrm{~mm}$, e a temperatura média anual oscila entre $19^{\circ} \mathrm{C}$ e $20^{\circ} \mathrm{C}$. O clima local, segundo a classificação de Köppen, é do tipo Cwa, tropical de altitude com verões quentes e chuvosos e invernos frios e secos. O solo da região apresenta a predominância de duas classes. No topo e nas encostas das elevações predominam os Latossolos e, nos terraços, predomina o Podzólico Vermelho-Amarelo Câmbico. A topografia local é acidentada com vales estreitos e úmidos. O relevo apresenta-se predominantemente forte, ondulado e montanhoso (ALMEIDA JÚNIOR, 1999; SOARES et al., 2010).

Entre 1942 e 1949, foram instaladas 12 parcelas experimentais de 600 a $1.000 \mathrm{~m}^{2}$ com onze espécies florestais, em espaçamento de $3 \times 2 m$. As espécies Aspidosperma polyneurom M. Arg. (peroba-rosa), Myracroduon urundeuva Fr.All. (aroeira-do-sertão), Hymenaea courbaril L. (jatobá), Lecythis pisonis Camb. (sapucaia), Aspidosperma parvifolium A.CD. (guatambu)

Revista Árvore, Viçosa-MG, v.35, n.5, p.1039-1051, 2011 
e Pterigota brasiliensis Fr. All. (pau-prata) foram plantadas em 1949. As espécies Tabebuia serratifolia (Vahl.) Nich. (ipê-amarelo) e Paratecoma peroba Kuhlm (peroba-do-campo) foram plantadas em 1945; Zeyheria tubrculosa (Vell.) Bur. (ipê-preto) e Pterogyne nitens Tull. (bilro) foram plantadas em 1943, e Balfourodendron riedelianum (Engl.) Engl. (pau-marfim) foi plantada em 1942.

Durante décadas, tratamentos silviculturais como capinas e roçados foram realizados anualmente. No entanto, a partir de 1980 as parcelas não sofreram mais limpeza, instalando-se, portanto, uma regeneração natural intensa sob os plantios e grande mortalidade devido à competição entre as árvores.

\subsection{Coleta e análise de dados}

Em cada parcela, foram tomadas as seguintes medidas das árvores: DAP (diâmetro com casca medido a 1,30 m do solo) e altura total (Ht) em metros (m).

As medidas de DAP foram utilizadas para caracterização da distribuição diamétrica, considerando classes de $2 \mathrm{~cm}$ de amplitude, e para seleção de três árvores-amostra por classe de diâmetro para realização do procedimento de cubagem rigorosa.

Os diâmetros com casca ao longo do fuste comercial das árvores foram obtidos com Pentaprisma de Wheeler, considerando seções de 2,0 m de comprimento. Os volumes dos fustes com casca das árvores foram obtidos pela aplicação sucessiva da equação de Smalian (SOARES et al., 2006).

Com os dados de DAP e altura total (Ht), foram ajustadas equações hipsométricas referentes aos seguintes modelos (CAMPOS; LEITE, 2006):

$$
\begin{aligned}
& \text { Modelo 1: } H t=\beta_{0} \cdot D A P^{\beta 1}+e \\
& \text { Modelo 2: } H t=\beta_{0} \cdot e^{\beta 1 / D A P}+e
\end{aligned}
$$

em que: $\beta_{0}$ e $\beta_{1}$ são os parâmetros do modelo; $e$ representa a exponencial; $\varepsilon$ é o erro aleatório; Ht é a altura total, em metros; e DAP é o diâmetro com casca a $1,30 \mathrm{~m}$ do solo, em $\mathrm{cm}$.

De posse dos dados da cubagem rigorosa, ou seja, dados de DAP, Ht e volumes dos fustes com casca das árvores, foram ajustadas equações de volumes referentes aos seguintes modelos (CAMPOS; LEITE, 2006; SOARES et al., 2006):
Modelo 1: Schumacher e Hall (1933):

$V=\beta_{0} \cdot D A P^{\beta 1} \cdot H t^{\beta 2}+\varepsilon$

Modelo 2: Spurr (1952): $V=\beta_{0^{.}}(D A P . H t)^{\beta 1}+\varepsilon(4)$

em que: $\beta_{0}$, $\beta_{1}$ e $\beta_{2}$ são os parâmetros do modelo; V é o volume do fuste com casca, em $\mathrm{m}^{3}$; $\varepsilon$ é o erro aleatório; DAP é o diâmetro com casca a 1,30 m do solo, em cm; e Ht é a altura total, em metros.

Também com os dados de cubagem rigorosa, foram ajustadas equações referentes aos seguintes modelos de taper:

Modelo 1: Demaerschalk (1972)

$\left(\frac{d}{D A P}\right)^{2}=10^{2 \beta_{0}} D A P^{\left(2 \beta_{1}-2\right)} L^{2 \beta_{2}} H t^{2 \beta_{3}}+\varepsilon$

Modelo 2: Ormerod (1973)

$$
\left(\frac{d}{D A P}\right)^{2}=\left[\frac{h-H t}{(H t-1,3)}\right]^{2 \beta_{1}}+\varepsilon
$$

Modelo 3: Biging (1984)

$$
d=D A P\left\{\beta_{1}+\beta_{2} \ln \left[1-(h / H t)^{1 / 3}\left(1-e^{-\beta 1 / \beta 2}\right)\right]\right\}+\varepsilon
$$

em que: $\beta_{0}, \beta_{1}, \beta_{2}$ e $\beta_{3}$ são os parâmetros do modelo; d é o diâmetro com casca na altura $h$, em cm; DAP é o diâmetro com casca a 1,30 m do solo, em cm; Ht é a altura total, em metros; h é a altura ao longo do fuste da árvore, em metros; L representa, $\mathrm{Ht}$ - h; ln é o logaritmo neperiano; $\varepsilon$ é o erro aleatório.

As equações foram ajustadas utilizando o algoritmo Gauss-Newton, por meio do software Statistica 8.0 (STATSOFT, 2008).

Para evitar julgamentos pessoais na seleção das melhores equações hipsométricas para cada espécie, foram utilizados os seguintes critérios de avaliação: a) medidas de precisão (coeficiente de determinação ' $\bar{R}^{2}$ ', e o erro-padrão da estimativa ' $S_{y . x}$ '); b) sinais associados aos coeficientes das equações; e c) análise gráfica dos resíduos em porcentagem (PAULA NETO, 1977).

Os mesmos critérios foram utilizados para a seleção das equações volumétricas e de taper, à exceção do coeficiente de determinação. Neste caso, foi utilizado o coeficiente de determinação ajustado $\left(\bar{R}^{2}\right)$, uma vez que as equações possuem diferentes números de variáveis independentes.

Revista Árvore, Viçosa-MG, v.35, n5, p.1039-1051, 2011 
O coeficiente de determinação e o coeficiente de determinação ajustado foram calculados pelos seguintes estimadores (KVÅLSETH, 1985):

$$
\begin{aligned}
& R^{2}(\%)=\left[1-\frac{\sum(y-\hat{y})^{2}}{\sum(y-\bar{y})^{2}}\right] \cdot 100 \\
& \bar{R}^{2}(\%)=\left[1-\left(\frac{n-1}{n-p-1}\right) \cdot\left(1-R^{2}\right)\right] \cdot 100
\end{aligned}
$$

em que: n é o número de observações; p é o número de variáveis independentes; y são os valores observados da variável dependente; $\hat{y}$ são os valores estimados das variáveis dependentes.

O erro-padrão da estimativa foi obtido pela seguinte expressão:

$$
S_{y \cdot x}=\sqrt{\frac{\sum(y-\hat{y})^{2}}{n-p-1}}
$$

\section{RESULTADOS}

\subsection{Caracterização dos plantios}

A espécie jatobá foi aquela que apresentou maior diâmetro e maior altura. As espécies peroba-rosa e pau-prata foram as que apresentaram maior número de indivíduos remanescentes (Tabela 1).

\subsection{Equações hipsométricas}

As equações hipsométricas apresentaram coeficientes de determinação $\left(\bar{R}^{2}\right)$ maiores que $60 \%$ (Tabela 2), com destaque para as espécies peroba-docampo, cujo $\bar{R}^{2}$ foi igual a 91,89\%; ipê-preto, com valor de $\bar{R}^{2}$ igual a $85,56 \%$; e peroba-rosa com valor de $\mathrm{R}^{2}$ igual a $85,5 \%$. Esses valores de foram obtidos utilizando o modelo 2 .

As equações referentes ao modelo 1 se ajustaram melhor aos dados observados de altura total somente para as espécies ipê-amarelo e aroeira-do-sertão. Para as demais espécies, as equações referentes ao modelo 2 foram as que se ajustaram melhor.

Os valores de erro-padrão de estimativa $\left(\mathrm{S}_{\mathrm{y} . \mathrm{x}}\right)$ ficaram entre 1,27 a 4,11 para o modelo 1 e entre 1,19 a 4,00 para o modelo 2. A espécie bilro foi a que apresentou pior ajuste de equação hipsométrica comparada com as outras espécies estudadas em ambos os modelos, com valores de $\mathrm{S}_{\mathrm{y} . \mathrm{x}}$ de 4,11 e 4,00 para o modelo 1 e 2, respectivamente. Em contrapartida, a espécie jatobá foi a que apresentou o melhor ajuste de equação hipsométrica para ambos os modelos em comparação com todas as espécies estudadas. Os valores de $\mathrm{S}_{\mathrm{y} . \mathrm{x}}$ para jatobá ficaram entre 1,27 e 1,19 para o modelo 1 e modelo 2 , respectivamente.

Apesar das equações hipsométricas para as 11 espécies apresentarem valores próximos entre si, nas duas estatísticas avaliadas, o modelo 2 foi ligeiramente superior em 9 das 11 espécies avaliadas. Com exceção

Tabela 1 - Características dendrométricas e número de indivíduos dos plantios na Mata da Silvicultura, Viçosa, Minas Gerais, Brasil.

\begin{tabular}{|c|c|c|c|c|c|c|c|c|}
\hline \multirow[b]{2}{*}{ Espécie } & \multicolumn{3}{|c|}{ Diâmetro $(\mathrm{cm})$} & \multicolumn{3}{|c|}{ Alturas totais (m) } & \multirow{2}{*}{$\begin{array}{c}\text { Altura } \\
\text { Comercial (m) }\end{array}$} & \multirow{2}{*}{$\begin{array}{c}\mathrm{N}^{\circ} \text { de } \\
\text { indivíduos }\end{array}$} \\
\hline & Mínimo & Médio & Máximo & Mínima & Média & Máxima & & \\
\hline$\overline{\text { Aroeira-do-sertão }}$ & 9,5 & 17,6 & 31,0 & 9,8 & 17,0 & 24,3 & 11,5 & 55 \\
\hline Bilro & 9,4 & 23,1 & 44,5 & 7,8 & 20,9 & 31,3 & 14,8 & 45 \\
\hline Guatambu & 5,3 & 18,0 & 42,0 & 7,0 & 19,2 & 28,5 & 14,4 & 84 \\
\hline Ipê-amarelo & 9,7 & 21,3 & 40,5 & 9,6 & 16,8 & 25,5 & 12,4 & 47 \\
\hline Ipê-preto & 10,4 & 19,6 & 37,0 & 6,8 & 16,6 & 26,0 & 13,4 & 20 \\
\hline Jatobá & 24,5 & 43,1 & 51,5 & 22,0 & 26,3 & 29,3 & 18,2 & 28 \\
\hline Pau-marfim & 7,0 & 19,6 & 41,4 & 5,2 & 19,3 & 27,3 & 12,7 & 69 \\
\hline Pau-prata & 7,0 & 24,3 & 48,6 & 8,3 & 20,8 & 31,5 & 16,6 & 100 \\
\hline Peroba-do-campo & 9,0 & 18,4 & 9,0 & 6,6 & 17,5 & 26,3 & 11,9 & 37 \\
\hline Peroba-rosa & 11,0 & 19,4 & 11,0 & 10,0 & 18,7 & 25,5 & 13,4 & 103 \\
\hline Sapucaia & 7,8 & 19,9 & 36,0 & 10,4 & 21,6 & 28,0 & 14,5 & 24 \\
\hline
\end{tabular}

Table 1 - Dendometric characteristics and number of individuals in plantings in Mata da Silvicultura, Viçosa, Minas Gerais, Brazil.

Revista Árvore, Viçosa-MG, v.35, n.5, p.1039-1051, 2011 
Tabela 2 - Avaliação das equações hipsométricas, volumétricas e de taper de acordo com seus coeficientes e suas medidas de precisão.

Table 2 - Evaluation of hypsometric, volumetric and taper equations, according to their coefficients and precision measures.

\begin{tabular}{|c|c|c|c|c|c|c|c|}
\hline \multirow[b]{3}{*}{ Espécie } & \multicolumn{7}{|c|}{ Equações hipsométricas } \\
\hline & \multirow[b]{2}{*}{ Modelo } & \multicolumn{4}{|c|}{ Coeficientes } & \multicolumn{2}{|c|}{ Medidas de precisão } \\
\hline & & $\mathrm{b}_{0}$ & $\mathrm{~b}_{1}$ & $\mathrm{~b}_{2}$ & $\mathrm{~b}_{3}$ & $\mathrm{R}^{2}(\%)$ & $\mathrm{S}_{\mathrm{y} . \mathrm{x}}$ \\
\hline \multirow[t]{2}{*}{ Ipê-amarelo } & 1 & 3,687691 & 0,496211 & - & - & 65,79 & 2,42 \\
\hline & 2 & 27,30911 & $-9,58412$ & - & - & 62,28 & 2,54 \\
\hline \multirow[t]{2}{*}{ Aroeira-do-sertão } & 1 & 3,613484 & 0,542622 & - & - & 68,64 & 2,17 \\
\hline & 2 & 30,03094 & $-9,40828$ & - & - & 67,28 & 2,21 \\
\hline \multirow[t]{2}{*}{ Peroba-do-campo } & 1 & 2,157994 & 0,694295 & - & - & 86,08 & 2,06 \\
\hline & 2 & 37,88383 & $-14,4673$ & - & - & 91,89 & 1,57 \\
\hline \multirow[t]{2}{*}{ Pau-prata } & 1 & 5,747979 & 0,398773 & - & - & 61,55 & 3,02 \\
\hline & 2 & 31,34440 & $-9,44009$ & - & - & 70,15 & 2,66 \\
\hline \multirow[t]{2}{*}{ Guatambu } & 1 & 4,434880 & 0,480998 & - & - & 76,30 & 2,84 \\
\hline & 2 & 29,85585 & $-8,08026$ & - & - & 76,63 & 2,82 \\
\hline \multirow[t]{2}{*}{ Bilro } & 1 & 2,673091 & 0,648624 & - & - & 67,11 & 4,11 \\
\hline & 2 & 41,40995 & $-15,1191$ & - & - & 68,92 & 4,00 \\
\hline \multirow[t]{2}{*}{ Ipê-preto } & 1 & 1,631752 & 0,786658 & - & - & 81,71 & 2,58 \\
\hline & 2 & 40,15851 & $-15,9457$ & - & - & 85,56 & 2,30 \\
\hline \multirow[t]{2}{*}{ Peroba-rosa } & 1 & 3,547764 & 0,539912 & - & - & 78,36 & 1,97 \\
\hline & 2 & 32,92539 & $-11,6279$ & - & - & 85,50 & 1,61 \\
\hline \multirow[t]{2}{*}{ Sapucaia } & 1 & 3,364302 & 0,577149 & - & - & 79,16 & 2,35 \\
\hline & 2 & 34,06659 & $-10,8704$ & - & - & 79,41 & 2,34 \\
\hline \multirow[t]{2}{*}{ Jatobá } & 1 & 8,491425 & 0,317333 & - & - & 65,80 & 1,27 \\
\hline & 2 & 36,68201 & $-11,5298$ & - & - & 69,93 & 1,19 \\
\hline \multirow[t]{3}{*}{ Pau-marfim } & 1 & 4,121420 & 0,495901 & - & - & 73,30 & 2,88 \\
\hline & 2 & 31,57432 & $-9,93978$ & - & - & 79,01 & 2,55 \\
\hline & \multicolumn{7}{|c|}{ Equações volumétricas } \\
\hline Espécie & Modelo & $\mathrm{b}_{0}$ & $\mathrm{~b}_{1}$ & $\mathrm{~b}_{2}$ & $\mathrm{~b}_{3}$ & $\bar{R}^{2}(\%)$ & $\mathrm{S}_{\mathrm{y} \cdot \mathrm{x}}$ \\
\hline \multirow[t]{2}{*}{ Peroba-do-campo } & 1 & 0,000048 & 2,123468 & 0,772306 & - & 98,10 & 0,0613 \\
\hline & 2 & 0,000032 & 1,010443 & - & - & 98,14 & 0,0606 \\
\hline \multirow[t]{2}{*}{ Ipê-preto } & 1 & 0,000024 & 2,086110 & 1,052790 & - & 98,80 & 0,0377 \\
\hline & 2 & 0,000024 & 1,045093 & - & - & 98,92 & 0,0357 \\
\hline \multirow[t]{2}{*}{ Jatobá } & 1 & 0,0000003 & 1,858675 & 2,653253 & - & 76,40 & 0,3695 \\
\hline & 2 & 0,000011 & 1,120225 & - & - & 80,38 & 0,3369 \\
\hline \multirow[t]{2}{*}{ Sapucaia } & 1 & 0,000093 & 1,864167 & 0,830800 & - & 92,56 & 0,0814 \\
\hline & 2 & 0,000076 & 0,921207 & - & - & 92,90 & 0,0795 \\
\hline Aroeira-do-sertão & 1 & 0,000378 & 2,586980 & $-0,442405$ & - & 87,96 & 0,0625 \\
\hline & 2 & 0,000126 & 0,853126 & - & - & 83,88 & 0,0723 \\
\hline Bilro & 1 & 0,001260 & 2,245434 & $-0,382858$ & - & 88,02 & 0,1809 \\
\hline & 2 & 0,000302 & 0,784934 & - & - & 83,93 & 0,2095 \\
\hline Guatambu & 1 & 0,000160 & 2,099119 & 0,359591 & - & 97,78 & 0,0589 \\
\hline & 2 & 0,000065 & 0,923264 & - & - & 97,31 & 0,0648 \\
\hline Ipê-amarelo & 1 & 0,000029 & 2,184931 & 0,872582 & - & 98,34 & 0,0509 \\
\hline & 2 & 0,000026 & 1,038914 & - & - & 98,27 & 0,0519 \\
\hline Peroba-rosa & 1 & 0,000084 & 2,233578 & 0,444409 & - & 98,88 & 0,0316 \\
\hline & 2 & 0,000023 & 1,037542 & - & - & 98,31 & 0,0388 \\
\hline Pau-prata & 1 & 0,000043 & 2,128779 & 0,832834 & - & 98,15 & 0,0918 \\
\hline & 2 & 0,000026 & 1,042081 & - & - & 98,03 & 0,0947 \\
\hline Pau-marfim & 1 & 0,000060 & 2,044059 & 0,745688 & - & 96,58 & 0,0768 \\
\hline & 2 & 0,000038 & 0,982722 & - & - & 96,52 & 0,0775 \\
\hline
\end{tabular}


Tabela 2 - Cont.

Table 2 - Cont.

\begin{tabular}{|c|c|c|c|c|c|c|c|}
\hline Espécie & Modelo & $\mathrm{b}_{0}$ & $\mathrm{~b}_{1}$ & $\mathrm{~b}_{2}$ & $\mathrm{~b}_{3}$ & $\bar{R}^{2}(\%)$ & $\mathrm{S}_{\mathrm{y} . \mathrm{x}}$ \\
\hline \multirow[t]{3}{*}{$\overline{\text { Ipê-amarelo }}$} & 1 & 0,229167 & 0,964566 & 0,802354 & $-0,901855$ & 84,99 & 3,4903 \\
\hline & 2 & - & 0,651339 & - & - & 86,03 & 3,3381 \\
\hline & 3 & - & 1,157399 & 0,297736 & - & 87,88 & 3,1230 \\
\hline \multirow[t]{3}{*}{ Peroba-do-campo } & 1 & 0,221797 & 1,012490 & 0,886559 & $-1,043197$ & 86,51 & 3,4231 \\
\hline & 2 & - & 0,862069 & - & - & 86,77 & 3,3612 \\
\hline & 3 & - & 1,131738 & 0,308989 & - & 88,52 & 3,1399 \\
\hline \multirow[t]{3}{*}{ Aroeira-do-sertão } & 1 & 0,075621 & 0,571067 & 1,228395 & $-0,781106$ & 62,43 & 3,7912 \\
\hline & 2 & - & 0,710230 & - & - & 74,55 & 3,0902 \\
\hline & 3 & - & 1,194987 & 0,362193 & - & 75,98 & 3,0120 \\
\hline \multirow[t]{3}{*}{ Pau-prata } & 1 & 0,258689 & 0,987194 & 0,573105 & $-0,732425$ & 90,26 & 3,0883 \\
\hline & 2 & - & 0,530194 & - & - & 90,98 & 2,9622 \\
\hline & 3 & - & 1,103060 & 0,240000 & - & 93,22 & 2,5712 \\
\hline \multirow[t]{3}{*}{ Guatambu } & 1 & 0,173279 & 0,920416 & 0,848163 & $-0,867180$ & 90,44 & 2,3917 \\
\hline & 2 & - & 0,779262 & - & - & 90,99 & 2,3100 \\
\hline & 3 & - & 1,172499 & 0,377498 & - & 93,25 & 2,0026 \\
\hline \multirow[t]{3}{*}{ Bilro } & 1 & 0,161454 & 0,959953 & 0,889554 & $-0,926701$ & 85,33 & 3,6370 \\
\hline & 2 & - & 0,729635 & - & - & 87,03 & 3,4014 \\
\hline & 3 & - & 1,199441 & 0,373179 & - & 89,63 & 3,0460 \\
\hline \multirow[t]{3}{*}{ Ipê-preto } & 1 & 0,161830 & 1,029927 & 0,654286 & $-0,788441$ & 95,07 & 1,5875 \\
\hline & 2 & - & 0,621653 & - & - & 95,05 & 1,5752 \\
\hline & 3 & - & 1,174937 & 0,333650 & - & 95,47 & 1,5112 \\
\hline \multirow[t]{3}{*}{ Peroba-rosa } & 1 & $-0,34058$ & 0,134075 & 1,465968 & $-0,219648$ & 61,78 & 5,2186 \\
\hline & 2 & - & 0,997446 & - & - & 82,57 & 3,5024 \\
\hline & 3 & - & 1,241009 & 0,498759 & - & 87,15 & 3,0133 \\
\hline \multirow[t]{3}{*}{ Jatobá } & 1 & 0,330650 & 0,969341 & 0,574657 & $-0,750611$ & 62,21 & 5,7887 \\
\hline & 2 & - & 0,497979 & - & - & 60,22 & 5,8962 \\
\hline & 3 & - & 1,143493 & 0,296756 & - & 60,95 & 5,8558 \\
\hline \multirow[t]{3}{*}{ Pau-marfim } & 1 & 0,187015 & 0,962291 & 0,670157 & $-0,749457$ & 88,49 & 2,9273 \\
\hline & 2 & - & 0,622623 & - & - & 88,29 & 2,9370 \\
\hline & 3 & - & 1,148645 & 0,317869 & - & 89,18 & 2,8278 \\
\hline \multirow[t]{3}{*}{ Sapucaia } & 1 & $-0,00040$ & 0,942105 & 0,937192 & $-0,836943$ & 82,91 & 3,6993 \\
\hline & 2 & - & 0,798527 & - & - & 83,89 & 3,5603 \\
\hline & 3 & - & 1,198831 & 0,397978 & - & 87,26 & 3,1753 \\
\hline
\end{tabular}

do ipê-amarelo e aroeira-do-sertão, deve ser dada preferência ao ajuste da equação hipsométrica referente ao modelo 2.

Na Figura 1, encontram-se os resíduos das equações hipsométricas que apresentaram maiores valores de $\bar{R}^{2}$ para cada espécie estudada. Estes resíduos estão distribuídos uniformemente, independente do tamanho das árvores, confirmando que as equações selecionadas através dos valores de $\bar{R}$ são as melhores equações hipsométricas para as espécies estudadas. Portanto, o modelo 1 foi o que melhor ajustou a relação hipsométrica para as espécies ipê-amarelo e aroeirado-sertão, enquanto o modelo 2 foi o que melhor ajustou essa relação hipsométrica para as espécies perobarosa, jatobá, sapucaia, guatambu, pau-prata, perobado-campo, ipê-preto e bilro.

\subsection{Equações de volume}

As equações de volume com casca para o fuste comercial das espécies estudadas apresentaram coeficientes de determinação ajustados $\left(\bar{R}^{2}\right)$ maiores que 76\% (Tabela 2). As espécies ipê-preto, perobarosa e ipê-amarelo foram as que obtiveram maiores valores de $\bar{R}^{2}$, sendo $98,92 \%$ utilizando o modelo 2 para ipêpreto, 98,88\% com o modelo 1 para peroba-rosa e 98,34\% utilizando o modelo 1 para o ipê-amarelo.

Revista Árvore, Viçosa-MG, v.35, n.5, p.1039-1051, 2011 

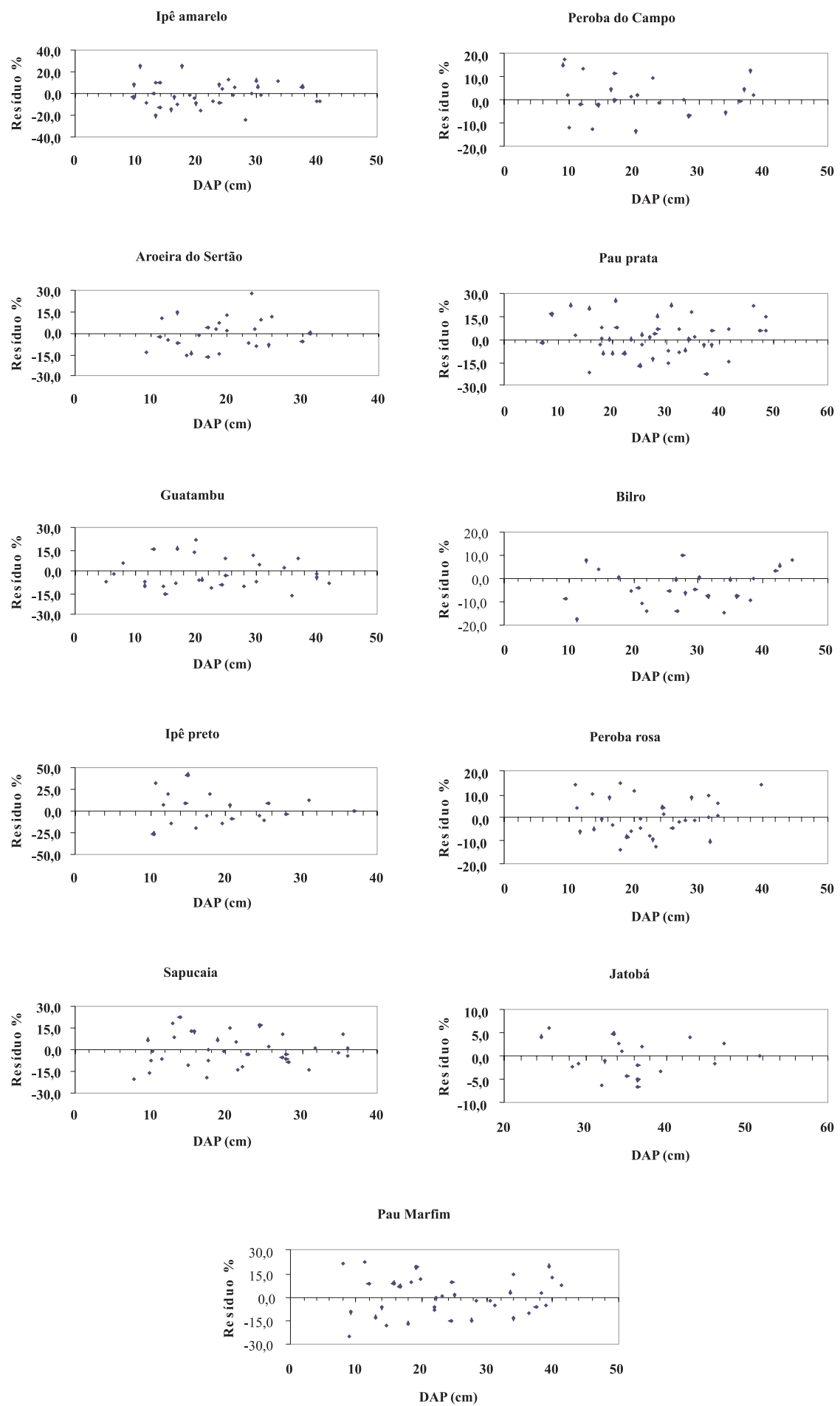

Figura 1 - Resíduos das equações hipsométricas para as espécies plantadas na Mata da Silvicultura, Viçosa, Minas Gerais, Brasil.

Figure 1 - Residuals of hypsometric equations for species in Mata da Silvicultura, Viçosa, Minas Gerais, Brazil. 
O erro-padrão de estimativa $\left(\mathrm{S}_{\mathrm{y} . \mathrm{x}}\right)$ variou de 0,0316 a 0,3695 para o modelo 1 de Schumacher e Hall (1933) e de 0,0357 a 0,3369 para o modelo 2 de Spurr (1952). A espécie jatobá e bilro foram as que apresentaram pior ajuste de equação volumétrica comparada com as outras espécies estudadas em ambos os modelos, com valores de $S_{y . x}$ para jatobá entre 0,3695 e 0,3369 para o modelo 1 e 2 , respectivamente, e valores de $\mathrm{S}_{\mathrm{y} . \mathrm{x}}$ para bilro entre 0,1809 e 0,2095 para o modelo 1 e 2, respectivamente. Em contrapartida, a espécie perobarosa foi a que apresentou o melhor ajuste de equação volumétrica para o modelo 1 em comparação com todas as espécies estudadas, apresentando valor de $\mathrm{S}_{\mathrm{y} . \mathrm{x}}$ igual a 0,0316, enquanto que o ipê-preto foi a espécie que apresentou melhor ajuste para o modelo 2, com valor de $\mathrm{S}_{\mathrm{y} \cdot \mathrm{x}} 0,0357$.

As equações referentes ao modelo 2 se ajustaram melhor aos dados observados de volume para as espécies peroba-do-campo, ipê-preto, jatobá e sapucaia, aroeirado-sertão e bilro. Para as demais espécies, as equações referentes ao modelo 1 foram as que se ajustaram melhor.

Com relação às espécies aroeira-do-sertão e bilro, foram observados sinais negativos de coeficientes associados à altura total (Ht) para essas duas espécies nas equações referentes ao modelo volumétrico 1 . Os valores encontrados foram - 0,442405 para aroeirado-sertão e -0,382858 para bilro. Os sinais negativos dos coeficientes associados à variável altura total (Ht) indicam erro de estimação destes coeficientes, uma vez que a variável Ht sempre apresenta correlação positiva com o volume da casca. Dessa forma, apesar do modelo 1 apresentar melhores medidas de precisão para estimar o volume da aroeira-do-sertão e bilro, o mesmo não foi o escolhido, devido apresentar inconsistência nos valores do coeficiente associado a $\mathrm{Ht}$.

O Cetec (1995) publicou equações de volume para diferentes formações florestais no estado de Minas Gerais e também para outros estados. Algumas equações para estimar o volume de galhos e o volume total da árvore em função do DAP e da altura comercial (Hc) apresentaram coeficientes negativos associados à variável altura. Neste trabalho especificamente, árvores com DAP pequenos e, consequentemente, volumes pequenos, apresentavam alturas comerciais maiores do que as árvores de maiores diâmetros. Assim, os sinais associados à variável altura estavam coerentes, diferentemente do resultado encontrado neste estudo.
Analisando a Figura 2, observa-se que os resíduos das equações previamente selecionadas estão uniformemente distribuídos, confirmando as equações previamente selecionadas como as melhores para estimar o volume com casca do fuste das espécies.

Semelhante ao ocorrido na equação hipsométrica, as equações volumétricas também apresentaram valores próximos entre si nas duas estatísticas avaliadas. A equação volumétrica referente ao modelo 1 , proposta por Schumacher e Hall, foi superior em 5 das 11 espécies e deve ser dada preferência ao ajuste desse modelo nas espécies guatambu, ipê-amarelo, peroba-rosa, pauprata e pau-marfim. O modelo 2, proposto por Spurr, foi superior às outras 6 espécies e deve ser dada preferência ao ajuste desse modelo para as espécies peroba-do-campo, ipê-preto, jatobá, sapucaia, aroeirado-sertão e birlo.

\subsection{Equações de taper}

De acordo com as medidas de precisão das equações de taper encontradas na Tabela 2, observa-se que o modelo 1, proposto por Demaerschalk (1972), foi o que melhor se ajustou aos dados observados para árvores da espécie jatobá. O modelo 3, proposto por Biging (1984), foi melhor para as demais espécies. Pode-se afirmar que as funções de afilamento para as demais espécies apresentaram uma razoável precisão na estimativa do diâmetro, com destaque para as espécies pau-prata, guatambu e ipê-preto, que apresentaram valores de de 93,22\%, 93,25\% e 95,47\%, respectivamente. Já as espécies jatobá e aroeira-do-sertão apresentaram piores ajustes nas equações de taper comparadas com todas as espécies, com valores de $\bar{R}^{2}$ 60,22\% para jatobá e 75,98\% para aroeira.

A Figura 3 apresenta os gráficos de resíduos das funções de taper. Esta figura demonstra, de maneira geral, que as equações tendem a estimar com maior precisão as árvores de maior tamanho, havendo maior erro das estimativas para as árvores de menor tamanho. Esta é uma tendência que normalmente ocorre quando se utilizam funções de afilamento, uma vez que a curva ajustada considera a forma média da árvore. Dessa forma, os diâmetros menores, por apresentarem valores absolutos menores, estão sujeitos a erros relativos maiores e, portanto, são mais difíceis de estimar com maior precisão. Uma alternativa plausível para algumas espécies, quando este fato ocorre com maior intensidade, é o uso de modelos segmentados, o que não foi o caso deste estudo. 


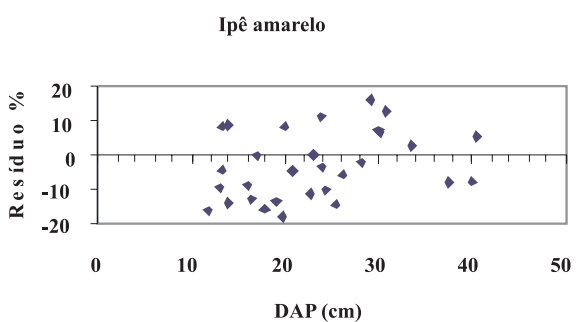

Aroeira do Sertão

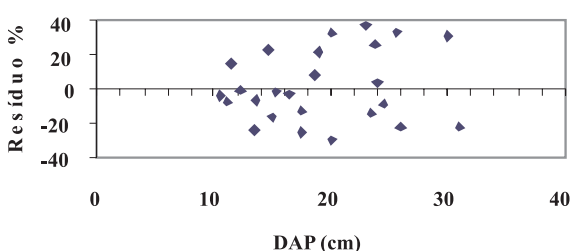

Guatambu

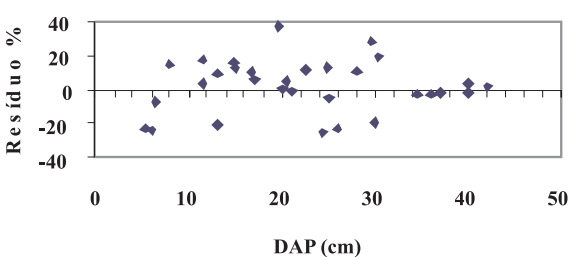

Ipê preto

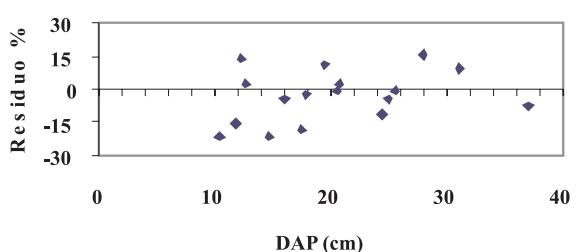

Sapucaia

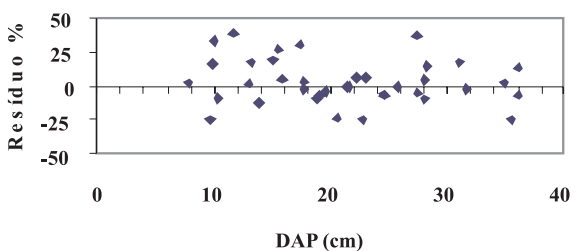

Peroba do Campo

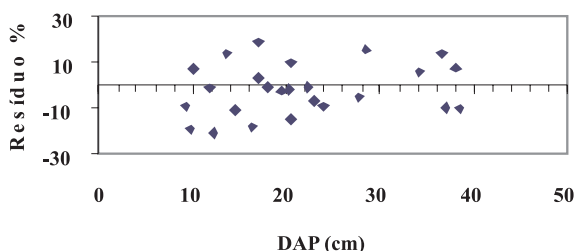

Pau prata

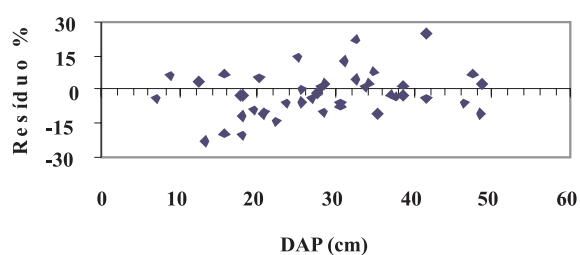

Bilro

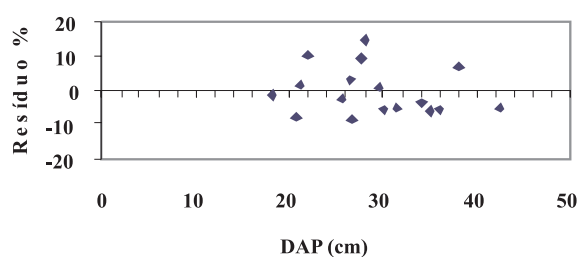

Peroba rosa

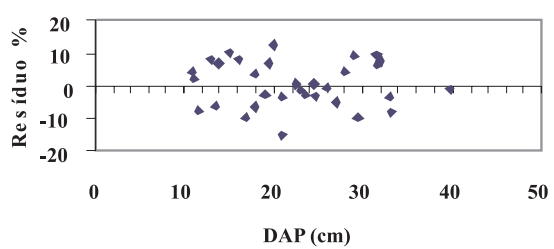

Jatobá

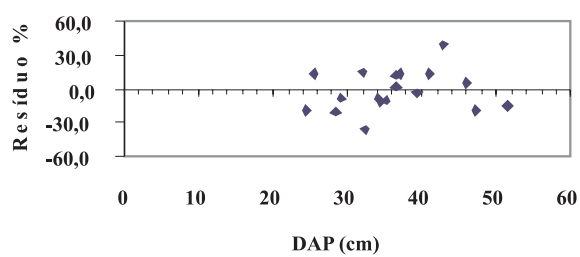

Figura 2 - Resíduos em porcentagem das equações volumétricas para as espécies plantadas na Mata da Silvicultura, Viçosa, Minas Gerais, Brasil.

Figure 2 - Residuals of volumetric equations for species in Mata da Silvicultura, Viçosa, Minas Gerais, Brazil. 
Ipê Amarelo

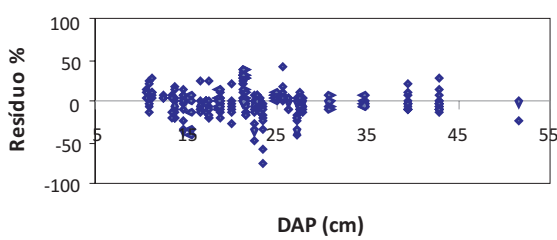

Aroeira do Sertão

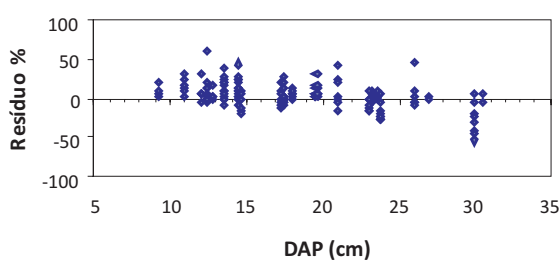

Guatambú

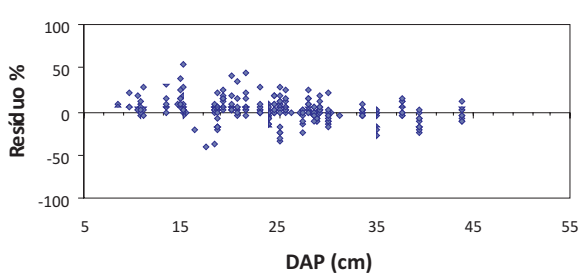

Ipê Preto

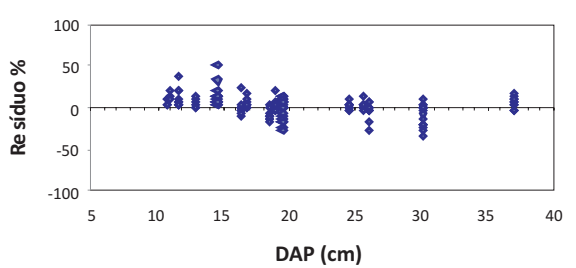

Sapucaia

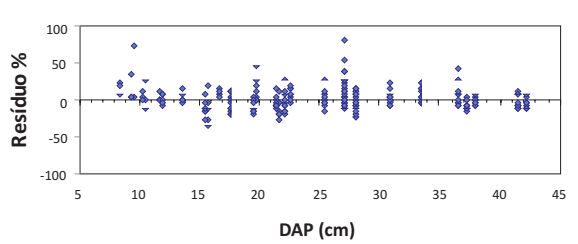

Peroba do Campo

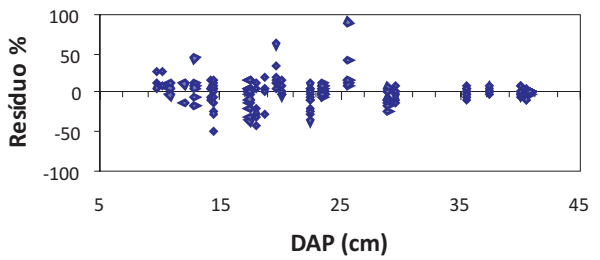

Pau Prata

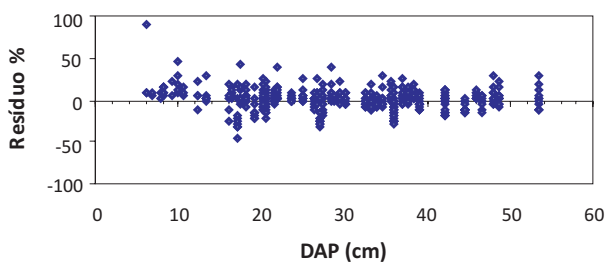

Bilro

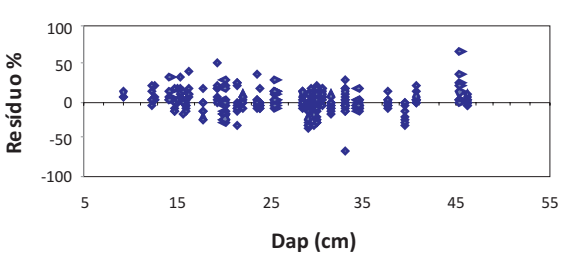

Peroba Rosa

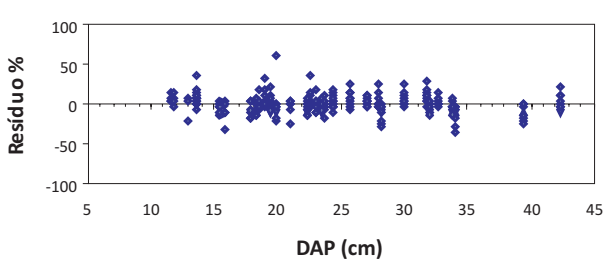

Jatobá

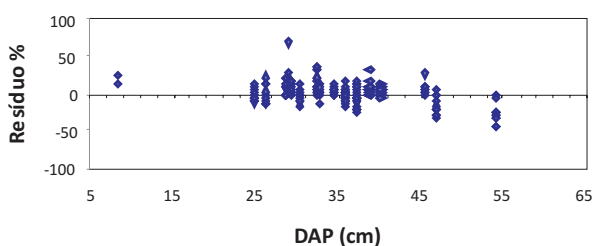

Figura 3 - Resíduos em porcentagem das equações de taper para as espécies plantadas na Mata da Silvicultura, Viçosa, Minas Gerais, Brasil.

Figure 3 - Residuals of taper equations for species in Mata da Silvicultura, Viçosa, Minas Gerais, Brazil.

Revista Árvore, Viçosa-MG, v.35, n.5, p.1039-1051, 2011 


\section{DISCUSSÃO}

Equações hipsométricas expressam a relação entre a altura das árvores com variáveis como idade, altura dominante, diâmetro médio, área basal e dap (ANDRADE et al., 2006). A equação hipsométrica depende, fundamentalmente, da espécie estudada, e a escolha correta da equação hipsométrica permite caracterizar o comportamento da altura das árvores nas quais esta variável não foi medida em diferentes locais ao longo do tempo, considerando que a determinação correta da altura permite inferir sobre a estrutura vertical de uma floresta inequiânea.

As equações hipsométricas ajustadas neste estudo apresentaram medidas de precisão semelhantes às encontradas nos ajustes de equações hipsométricas para florestas naturais e para espécies isoladas. Andrade et al. (2006) ajustaram equações hipsométricas para um fragmento de Floresta Estacional Semidecidual na região norte do estado do Rio de Janeiro e encontraram $\bar{R}^{2}$ entre 0,61 e 0,85 , valores semelhantes aos encontrados neste estudo.

Coraiola e Netto (2003) também ajustaram equações hipsométricas para uma Floresta Estacional Semidecidual no sul do estado de Minas Gerais, encontrando $\bar{R}^{2}$ maiores que 0,91 . Neste caso, os autores estratificaram a floresta em classes de altura, melhorando significativamente o ajuste das equações.

Tonini e Schwenberg (2006) testaram oito modelos hipsométricos para plantios de Acacia mangium em Roraima, sendo que equações referentes aos oito modelos testados apresentaram $\bar{R}^{2}$ entre 0,41 e 0,98. Tonini et al. (2008) avaliaram seis modelos hipsométricos para populações nativas de castanheira-do-brasil (Berthollettia excelsa Bonpl.) e encontraram entre 0,54 e 0,70, inferiores aos encontrados neste estudo.

Com relação às equações volumétricas, Tonini et al. (2005) avaliaram dezenove modelos volumétricos lineares para estimar o volume de plantios homogêneos de andiroba (Carapa guianensis Aubl), castanha-dobrasil (Bertholletia excelsa Bonpl.), ipê-roxo (Tabebuia avellanedae Lorenz ex Griseb) e jatobá (Hymenea courbaril L.), encontrando para as equações selecionadas coeficientes de determinação ajustado entre 91 e 95\%. Apesar dos modelos testados neste estudo serem diferentes dos modelos testados por Tonini et al. (2005), somente as espécies jatobá, aroeira-dosertão e bilro apresentaram valores de $\bar{R}^{2}$ menores do que os encontrados no referido artigo.

Os modelos de taper foram desenvolvidos para serem aplicados em florestas plantadas puras e florestas naturais mistas, em países de clima temperado, para a determinação dos volumes comercial e total, da altura comercial e do diâmetro superior (DEMAERSCHALK, 1972; BIGING, 1984). No Brasil, a maioria dos modelos de taper estão relacionados a florestas equiâneas puras (SCHNEIDER et al., 1996), sendo raros os trabalhos que estudam a forma da árvore por meio de funções de taper para espécies nativas. Um exemplo é o trabalho de Chichorro et al. (2003), que encontraram $\bar{R}^{2}$ maior que 93\% para modelos de taper com o objetivo de quantificar múltiplos produtos da madeira da Mata Atlântica no Espírito Santo. Neste trabalho, apenas as equações para as espécies ipê-preto, pau-prata e guatambu apresentaram coeficientes de determinação ajustado superiores a este valor. Pode-se dizer que estes resultados podem ser esperados, uma vez que a forma de espécies nativas com praticamente nenhum grau de domesticação ou melhoramento tendem a ser mais irregulares e heterogêneas, tornando mais difícil a sua estimação. Com base nisso, os resultados encontrados nesse estudo foram satisfatórios em termos de precisão, porém não se exclui a necessidade de novas pesquisas sobre o assunto.

\section{CONCLUSÕES}

Após as análises, pode-se concluir que:

O modelo hipsométrico 2 foi o que melhor se ajustou aos dados de nove espécies (peroba-do-campo, pauprata, guatambu, birlo, ipê-preto, peroba-rosa, sapucaia, jatobá e pau-marfim) das onze avaliadas;

O modelo volumétrico de Spurr (1952) foi o que se ajustou melhor aos dados de volume do fuste com casca para seis espécies (peroba do campo, ipê-preto, jatobá, sapucaia, aroeira-do-sertão e birlo), enquanto o modelo de Schumacher e Hall (1933) se ajustou melhor a cinco espécies (guatambu, ipê-amarelo, peroba-rosa, pau-prata e pau-marfim).

O modelo de taper de Biging (1984) foi superior em dez das onze espécies avaliadas (exceto jatobá), para descrever o perfil do tronco.

Revista Árvore, Viçosa-MG, v.35, n5, p.1039-1051, 2011 


\section{REFERÊNCIAS}

ALMEIDA JÚNIOR, J. S., Florística e fitossociologia de fragmentos da floresta estacional semidecidual, Viçosa, Minas Gerais. 1999. 148f. Dissertação (Mestrado em Ciência Florestal) Universidade Federal de Viçosa, Viçosa, MG, 1999.

ANDRADE, V. C. L. et al. Influência de três sistemas de amostragem na estimativa da relação hipsométrica e do volume de árvores em um fragmento de Mata Atlântica. Scientia Forestalis, n.70, p.31-37, 2006.

ARAujo, L. V. C.; LEITE, J. A. N.; PAES, J. B. Estimativa da produção de biomassa de um povoamento de Jurema-preta (Mimosa tenuiflora (WILLD). Pioret.) com cinco anos de idade. Biomassa e Energia, v.1, n.4, p.347-352, 2004.

ASSIS, A. L. Acuracidade de modelos polinomiais segmentados para estimativa de diâmetros e volumes ao longo do fuste de Pinus taeda. Revista Árvore, v.26, n.1, p.59-72, 2002.

BATISTA, J. L. F.; MARQUESINI, M.; VIANA, V. M. Equações de volume para árvores de caxeta (Tabebuia cassinoides) no Estado de São Paulo e sul do Estado do Rio de Janeiro. Scientia Forestalis, n.65, p.162-175, 2004.

BIGING, G. S. Taper equations for second mixedconifers of Northean California. Forest Science, v.30, n.4, p.1103-1117, 1984.

CAMPOS, J. C. C.; LEITE, H. G. Mensuração florestal: perguntas e respostas. Viçosa, MG: Universidade Federal de Viçosa, 2006. 407p.

FUNDAÇÃO CENTRO TECNOLÓGICO DE MINAS GERAIS - CETEC. Determinação de equações de volumétricas aplicáveis ao manejo sustentável de florestas nativas no estado de Minas Gerais e outras regiões do país. Belo Horizonte: 1995. 295p.

CHICHORRO, F.; RESENDE, J. L. P.; LEITE, H. G. Equações de volume e de taper para quantificar multiprodutos da madeira em floresta Atlântica. Revista Árvore, v.27, n.6, p.799-809, 2003.
CORAIOLA, M.; PÉLLICO NETTO, S. Análise da estrutura dimensional de uma floresta estacional semidecidual localizada no município de CássiaMG: estrutura volumétrica. Revista

Acadêmica: Ciências Agrárias e Ambientais, v.1, n.4, p.11-24, 2003.

DEMAERSCHALK, J. P. Converting volume equations to compatible taper equations. Forest Science, v.18, n.3, p.241-245, 1972.

FERNANDES, N. P.; JARDIM, F. C. S.; HIGUCHI, $\mathrm{N}$. Tabelas de volume para floresta de terra firme da Estação Experimental de Silvicultura Tropical. Acta Amazônica, v.13, n.3/4, p.537-545, 1983.

GUJARATI, D. N. Econometria básica. 3.ed. São Paulo: Pearson Education do Brasil, 2000. 846p.

KVÅLSETH, T. O. Cautionary note about $\mathrm{R}^{2}$. The American Statistician, v.39, n.4, p.279-285, 1985.

LIMA, F. S. Análise de funções de taper destinadas à avaliação de multiprodutos de árvores de Pinus elliottii. 1986. 77f. Dissertação (Mestrado em Ciência Florestal) - Universidade Federal de Viçosa, Viçosa, MG, 1986.

MEIRA NETO, J. A. A.; MARTINS, F. R. Estrutura da Mata da Silvicultura, uma floresta estacional semidecidual no município de Viçosa - MG. Revista Árvore, v.24, n.2., p.151-160, 2000.

ORMEROD, D. W. A simples bole model. The Forestry Chronicle, v.49, n.3, p.136-138, 1973.

PAULA NETO, F. Tabelas de volumétricas com e sem casca para Eucalyptus saligna. Revista Árvore, v.1, n.1, p.31-54, 1977.

PINHEIRO, G.; GARRIDO, L.; GARRIDO, M. Determinação de equações de volume comercial para cinco espécies de cerrado. Boletim Técnico do Instituto Florestal, n.38, p.1-9, 1985.

SCHNEIDER, P. R. et al. Forma de tronco e sortimentos de madeira de Eucalyptus grandis Maiden para o estado do Rio Grande do Sul. Ciência Florestal, v.47, n.1, p.79-88, 1996. 
SCHUMACHER, F.; HALL, F. X. Logarithmic expression of timber-tree volume. Journal of Agricultural Research, v.47, p.719-734, 1933.

SCOLFORO, J. R. S.; LIMA, J. T.; SILVA, S. T. Equações de biomassa e volume para cerrado sensu strictu. In: CONGRESSO FLORESTAL PANAMERICANO, 1.; CONGRESSO FLORESTAL BRASILEIRO, 7., 1993, Curitiba. Anais... Curitiba: 1993. p.508-510.

SCOLFORO, J. R. S.; MELLO, J. M.; LIMA, C. S. A. Obtenção de relações quantitativa para estimativa de volume do fuste em Floresta Estacional Semidecídua Montana. Revista Cerne, v.1, n.1, p.123-134, 1994.

SCOLFORO, J. R. S. et al. Estimativas de volume, peso seco, peso de óleo e quantidade de moirões para candeia (Eremanthus erytropappus).

Revista Cerne, v.10, n.1, p.87-102, 2004.

SILVA, J. N. M.; ARAÚJO, S. M. Equação de volume para árvores de pequeno diâmetro na Floresta Nacional do Tapajós. Boletim de Pesquisa Florestal, v.8, n.9, p.16-25, 1984.

SILVA, J. N. M.; CARVALHO, M. S. P. Equações de volume para uma floresta secundária no Planalto do Tapajós, Belterra, PA. Boletim de Pesquisa Florestal, v.8, n.9, p.1-15, 1984.

SILVA, G. F. Alternativas para estimar o volume comercial em árvores de eucalipto. 1996. 87f. Dissertação (Mestrado em Ciência Florestal) - Universidade Federal de Viçosa, Viçosa, MG, 1996.

SOARES, C. P. B., PAULA NETO, F., SOUSA, A. L. Dendrometria e inventário Florestal. Viçosa, MG: Universidade Federal de Viçosa, 2006. 276p.

SOARES, C. P. B.; SILVA, G. F.; MARTINS, F. B. Influence of sections lengths on volume determination in eucalyptus trees. Cerne, v.16 n. 2. p.155-162, 2010
SOUZA, A. L.; JESUS, R. M. Equações de volume comercial e fator de forma para espécies da mata atlântica ocorrentes na reserva florestal da Companhia Vale do Rio Doce, Linhares, ES. Revista Árvore, v.15, n.3, p.257-276, 1991.

SPURR, S. Forest inventory. New York: The Ronald Press, 1952. 476p.

STATSOFT INSTITUTE CORPORATION. Statistica for Windows: Version 8.0. Tulsa: 2008.

TONINI, H.; ARCO-VERDE, M. F.; SÁ, S. P. P. Dendrometria de espécies nativas em plantios homogêneos no estado de Roraima - Andiroba (Carapa guianensis Aubl), Castanha do Brasil (Bertholletia excelsa Bonpl.), Ipê-roxo (Tabebuia avellanedeae Lorentz ex Griseb) e Jatobá (Hymeneaea courbaril L.). Acta Amazônica, v.35, n.3, p.353-362, 2005.

TONINI, H.; SCHWENGBER, L. A. M. Equações hipsométricas e volumétricas para Acacia mangium Willd em Roraima. Ambiência, v.2, n.2, p.155-165, 2006.

TONINI, H.; COSTA, P.; KAMINSKI, P. E. Estrutura e produção de duas populações nativas de Castanheira-do-Brasil (Berthollettia excelsa O. Berg) em Roraima. Floresta, v.38, n.3, 2008.

YARED, J. A. G.; LEITE, H. G.; SILVA, R. R. F. Volumetria e fator de forma de morototó (Didymopanax morototonii) sob diferentes espaçamentos. In: CONGRESSO FLORESTAL PANAMERICANO, 1.; CONGRESSO FLORESTAL BRASILEIRO, 7., Curitiba, PR. Anais ... Curitiba: 1993. p.570-573.

ZAKIA, M. J. B.; PAREYN, F.; RIEGELHAUPT, E. Equações de peso e volume para oitos espécies lenhosas nativas do semiárido, RN. (Circular técnica PNUD/FAO/BRA/ 87/007), Mossoró:1990. p. 1-5. 
